J. Vet. Med. B 52, 367-371 (2005)

(C) 2005 The Authors

Journal compilation (C) 2005 Blackwell Verlag, Berlin

ISSN 0931-1793

Unit of Veterinary Epidemiology, Faculty of Veterinary Medicine, Department of Reproduction, Obstetrics and Herd Health, Ghent University, Merelbeke, Belgium

\title{
Evaluation of the epidemiological importance of classical swine fever infected, E2 sub-unit marker vaccinated animals with RT-nPCR positive blood samples
}

\author{
J. Dewulf ${ }^{1,4}$, F. Koenen ${ }^{2}$, S. Ribbens ${ }^{1}$, A. Haegeman ${ }^{2}$, H. Laevens ${ }^{3}$ and A. De Kruif ${ }^{1}$
}

Addresses of authors: ${ }^{1}$ Unit of Veterinary Epidemiology, Faculty of Veterinary Medicine, Department of Reproduction, Obstetrics and Herd Health, Ghent University, Salisburylaan 133, B-9820 Merelbeke, Belgium; ${ }^{2}$ Veterinary and Agrochemical Research Centre (CODA/CERVA), Section of Modelisation of Epizootic Diseases, Groeselenberg 99, B-1180 Brussels, Belgium; ${ }^{3}$ Veterinary and Agrochemical Research Centre (CODA/CERVA), Coordination Center for Veterinary Diagnostics, Groeselenberg 99, B-1180 Brussels, Belgium; ${ }^{4}$ Corresponding author: Tel: + 32926475 43; fax: + 32926475 34; E-mail: jeroen.dewulf@ugent.be

With 2 tables

Received for publication July 6, 2005

\begin{abstract}
Summary
It has been demonstrated that pigs that have been double vaccinated with an E2 sub-unit marker vaccine and that are infected with classical swine fever virus (CSFV) through a natural contact infection may react positive in a CSFV detecting RT-nPCR test, whereas no virus could be isolated by using the conventional virus isolation (VI) technique. To evaluate whether these vaccinated and infected pigs may spread the virus, three experiments were set up. In the first, susceptible pigs were inoculated with serum originating from vaccinated RT-nPCR positive pigs. In the second, vaccinated RT-nPCR positive pigs were brought into contact with sentinel animals. In the third, vertical transmission was evaluated in RT-nPCR positive vaccinated pregnant gilts. In the first two experiments, no proof of virus transmission was found, whereas in the third vertical transmission was observed. The conclusion is that in vaccinated pigs that are positive in RTnPCR but negative in VI, the level of circulating virus is probably not high enough for horizontal transmission, whereas vertical transmission of the virus is possible.
\end{abstract}

\section{Introduction}

Classical swine fever (CSF) is a contagious pig disease caused by the CSF virus (CSFV), which belongs to the genus pestivirus of the Flaviviridae family. During the 90s, several huge CSF outbreaks in Europe have exemplified that, in densely populated livestock areas and in absence of vaccination, outbreaks can only be controlled successfully by extensive pre-emptive culling in the neighbourhood of infected herds (Koenen et al., 1996; Meuwissen et al., 1999). This massive killing and destruction of mostly non-infected animals is increasingly perceived as wasteful and ethically unacceptable (Terpstra, 1998). Moreover, emergency vaccination, in combination with basic control measures could be an alternative.

In recent years, progress has been made in the development of marker vaccines and accompanying discriminatory diagnostic tests (Moormann et al., 2000). Currently, there are E2 sub-unit marker vaccines commercially available.

It has been demonstrated that an E2 sub-unit marker vaccine is able to protect vaccinated pigs against the clinical symptoms of a natural CSF infection, but not to prevent the infection as such (Dewulf et al., 2000, 2002). Some of these sub-clinically infected, vaccinated pigs only develop antibodies that can be detected with the differential ELISA, without a detectable viraemia. Others react positive in the reverse transcriptase (RT) nested polymerase chain reaction (nPCR) test but no virus can be isolated by using the conventional virus isolation (VI) technique (Dewulf et al., 2000, 2002). To be able to assess the effect of vaccination on the spread of the disease, it is critical to know whether these RT-nPCR positive pigs are infectious themselves and can spread the disease.

The aim of the experiments was to evaluate whether these vaccinated and infected RT-nPCR positive pigs can transmit the virus vertically or horizontally.

\section{Materials and Methods}

Experiment 1

Animals

Five clinically healthy, conventional weaner pigs (stress negative Belgian landrace) of mixed sex, originating from a known bovine viral diarrhoea (BVD) and CSF free herd were used. These animals were checked for the absence of BVD and CSF antigen and antibodies at their arrival. The detection of BVD and CSF virus was done by VI. The detection of BVD and CSF antibodies was done by virus neutralization (VN).

\section{Experimental design}

From a stock of serum samples, stored at $-80^{\circ} \mathrm{C}$, collected during a previous experiment with marker vaccinated pigs, five samples were selected. These were RT-nPCR positive and VI negative samples and had been collected from naturally infected marker-vaccinated pigs. The experiment from which these samples originate was described in detail by Dewulf et al. (2000). After thawing, the samples were analyzed again using RT-nPCR and VI. All were positive on RT-nPCR and negative on VI.

Ten days after the arrival of animals in the isolation unit (acclimatization period), the serum samples were inoculated by deep intramuscular injection in five fully susceptible pigs. Subsequently, the inoculated pigs were followed for 14 days (observation period) and blood sampled every other day 
during this period. Blood samples were analysed using VI and RT-nPCR.

\section{Sample collection and clinical examination}

During the acclimatization period, clotted and heparinized blood samples were collected from all pigs upon arrival, and 1 week later. From the day of inoculation onwards, pigs were blood sampled every 2 days during 2 weeks. After 2 weeks all pigs were euthanized and tissue samples (tonsil, kidney, spleen, heart and liver) were collected.

During the observation period, pigs were clinically inspected every day. Also, the rectal temperature was monitored daily.

\section{Sample analyses}

For VI, $100 \mu$ of whole blood was inoculated in duplicate onto a non-confluent monolayer of $\mathrm{PK}_{15}$ cells cultured in multiwell plates (24-wells/plate). For VI in tissue samples, $1 \mathrm{~cm}^{3}$ of each organ was homogenized into $9 \mathrm{ml}$ minimal essential medium (MEM) and ground with an ultra-Turrax. After centrifugation for $10 \mathrm{~min}$ at $4000 \mathrm{~g}, 300 \mu \mathrm{l}$ of the supernatant was inoculated in duplicate onto a non-confluent monolayer of $\mathrm{PK}_{15}$ cells cultured in multiwell plates (24-wells/plate). After $48 \mathrm{~h}$, the cells were fixed with isopropanol and stained with a polyclonal fluorescein-conjugated anti-CSFV immunoglobulin. Whenever a sample was positive in RT-nPCR without being positive in VI, three additional cell culture passages were done.

The single tube RT-nPCR was performed as described by McGoldrick et al. (1999). First, the RNA was extracted from serum samples using the QIAamp Viral RNA Kit (Qiagen, Ven lo, the Netherlands) according to the instructions of the manufacturer. RNA samples were stored at $-80^{\circ} \mathrm{C}$ before analysis. The combined reverse transcription and first amplification was carried out in a total volume of $50 \mu \mathrm{l}$ containing: $2 \mu \mathrm{l}$ of RNA, $20 \mathrm{~mm}$ Tris- $\mathrm{HCl}$ ( $\mathrm{pH} 8.4$ ), $50 \mathrm{~mm} \mathrm{KCl,} 6 \mathrm{~mm} \mathrm{MgCl}$, $0.37 \mathrm{~mm}$ of each dNTP, $5 \mathrm{pmol}$ of each primer (A11/A14), $0.2 \%$ Triton X-100, 2.5 U Taq (Invitrogen, Merelbeke, Belgium), $100 \mathrm{U}$ M-MLV (Invitrogen) and $10 \mathrm{U}$ RNase OUT inhibitor (Invitrogen). The water phase was overlaid with two drops of mineral oil. The in vitro amplification was performed in a GeneAmp 2400 system (Perkin Elmer, Wellesley, MA, USA) using the following thermal profile: 1 cycle of $42^{\circ} \mathrm{C}$ for $30 \mathrm{~min}$; 1 cycle of $95^{\circ} \mathrm{C}$ for $3 \mathrm{~min} ; 20$ cycles of $94^{\circ} \mathrm{C}, 60^{\circ} \mathrm{C}$ and $72^{\circ} \mathrm{C}$ for $1 \mathrm{~min}$ each respectively. The subsequent second amplification was achieved by inverting the tubes and thereby dissolving the air-dried pellet in the caps, containing $20 \mathrm{pmol}$ of the appropriate primers (V324/V326), $10 \mathrm{~mm}$ of each dNTP and 1.25 U Taq (Invitrogen). The second amplification profile was as follows: 30 cycles of $94^{\circ} \mathrm{C}, 60^{\circ} \mathrm{C}$ and $72^{\circ} \mathrm{C}$ for 1 min each; 1 cycle of $72^{\circ} \mathrm{C}$ for $10 \mathrm{~min}$. The amplified fragments were verified by electrophoresis on $2 \%$ agarose gels and visualized under UV light.

The virus neutralization is based on the NPLA test as described by Holm-Jensen (1981), using the Alfort187 strain.

\section{Experiment 2}

\section{Animals}

Thirty-one clinically healthy, conventional weaner pigs (stress negative Belgian landrace) of mixed sex were used. They fulfilled the same criteria as the animals of experiment 1 .

\section{Virus}

The isolate used for challenge was obtained originally from the first infected herd of the 1993-1994 Belgian CSF epidemic. The isolate was verified to be free of African-swine-fever-virus and BVD virus by means of VI and RT-nPCR. By using a panel of monoclonal antibodies, the virus was characterized as antigenically similar to an isolate known as the 'souche Lorraine' (Koenen and Lefebvre, 1994), which can be described as a moderately virulent strain following the clinical and pathological criteria proposed by Floegel-Niesmann et al. (2003). The virus was cultivated on $\mathrm{PK}_{15}$ cells and two passages were carried out. The titre was $10^{3}$ median tissue-culture-infective doses $\left(\right.$ TCID $\left._{50} / \mathrm{ml}\right)$. Pigs were challenged with a dose of $2 \mathrm{ml}$ intramuscular.

\section{Vaccine and vaccination}

The vaccine used was a sub-unit marker vaccine (Porcilis ${ }^{\circledR}$ Pesti; Intervet, Boxmeer, the Netherlands) consisting of the E2 glycoprotein of the CSF virus, strain Alfort/Tübingen. The glycoprotein was produced by means of a baculovirus expressing the protein in insect cells (Hulst et al., 1993). Pigs were vaccinated intramuscularly with a single vaccine dose of $2 \mathrm{ml}$.

\section{Experimental design}

Twenty-one conventional pigs were randomly allocated to two separated pens (10 and 11 pigs in pens 1 and 2 respectively). After an acclimatization period of 10 days, seven and eight pigs were randomly selected from pens 1 and 2, respectively, and were vaccinated. Four weeks later, these same pigs were booster vaccinated. Two weeks after the booster vaccination, the three remaining unvaccinated pigs in each pen were inoculated with the CSFV. The vaccinated pigs [referred to as contact pigs $1\left(\mathrm{C}_{1}\right)$ ] remained in the same pen with unvaccinated, inoculated pigs for 16 days.

After these 16 days, the unvaccinated inoculated pigs were euthanized and the $\mathrm{C}_{1}$ pigs were moved to two new pens in a separated compartment where they were, in each pen, housed together with five non-vaccinated contact pigs $\left(\mathrm{C}_{2}\right)$. During an observation period of 32 days, it was evaluated whether the sentinel animals $\left(\mathrm{C}_{2}\right)$ became infected. After the observation period, all $\mathrm{C}_{1}$ and $\mathrm{C}_{2}$ pigs were euthanized and tissue samples were collected.

\section{Sample collection and clinical examination}

During the acclimatization period, clotted and heparinized blood samples were collected from all pigs upon arrival, and 1 week later. During the vaccination period, blood samples were collected weekly. From the day of inoculation onwards, the inoculated as well as the $\mathrm{C}_{1}$ pigs were blood sampled every other day. During the observation period, the $\mathrm{C}_{1}$ and $\mathrm{C}_{2}$ pigs were also sampled every other day. At the end of the experiment, all pigs were euthanized and tissue samples (tonsil, kidney, spleen, heart and liver) were collected.

From the moment of inoculation onwards, clinical inspection and rectal temperature monitoring was performed on a daily bases. 


\section{Sample analyses}

Blood samples were analysed using VI and RT-nPCR (cf. experiment 1). The differential ELISA test used was the Chekit ${ }^{\circledR}$ CSF-Marker (Dr Bommeli AG, Switzerland).

\section{Experiment 3}

The material and methods of experiment 3 is described in detail in Dewulf et al., 2002.

The experimental design, which is of relevance for this paper, is explained briefly: eight pregnant, double vaccinated (E2 sub-unit marker vaccine) gilts were housed together with two unvaccinated CSF infected gilts. All sows were housed in individual sow boxes in which direct contact was only possible between neighbouring animals. Each unvaccinated infectious gilt (seeder animal) was surrounded at both sides by two vaccinated pregnant sows. The seeder sows were infected with CSFV 46 days after the booster vaccination of the vaccinated contact sows. At that moment, the sows were around day 40 of the gestation. In the 70-days post-inoculation (pi) period, all gilts were blood sampled every 3 days. Samples were analysed using VI and RTnPCR. One week before the end of gestation, the gilts were euthanized and tissue samples were collected from gilts and all foetuses.

All experiments were approved by the ethical committee of the Veterinary and Agrochemical Research Centre.

\section{Results}

\section{Experiment 1}

All blood samples collected from the five inoculated pigs remained negative in VI as well as in RT-nPCR during the whole observation period. Also, all tissue samples collected after euthanasia were negative in VI.

\section{Experiment 2}

The results of experiment 2 are summarized in Table 1 .

On the day of inoculation, all vaccinated pigs had virus neutralizing antibodies.

All experimentally inoculated pigs (seeders) became viraemic in VI from day- 4 or -6 pi onwards. Using RT-nPCR, the samples became positive from day 2 or 4 pi. In all pigs, the viraemia remained present until they were euthanized on day16 pi. They also developed clear clinical symptoms such as conjunctivitis, erythema and ataxia. Also, a clear rise of the rectal temperature was observed.

Twelve of 15 vaccinated contact pigs $\left(C_{1}\right.$; six in each pen $)$ seroconverted against the wild-type virus. None of the 12 infected vaccinated pigs developed a detectable viraemia in VI at any point during the experiment. Using RT-nPCR, positive blood samples were found in four pigs. Each positive RTnPCR result was sequenced and confirmed as being the same isolate obtained from the infected seeder pigs. For those samples that were positive in RT-nPCR, but remained negative in VI, three additional cell culture passages were performed. All remained negative.

None of the $\mathrm{C}_{1}$ pigs developed any clinical symptom or fever.

In the $\mathrm{C}_{2}$ pigs, all blood samples remained negative in VI, RT-nPCR, as well as virus neutralization during the whole observation period. Neither were there any clinical symptoms or rectal temperature rises.

\section{Experiment 3}

The results of experiment 3 are described in detail in Dewulf et al. (2002). Only those results relevant to this study are reported, completed with some additional data (Table 2).

Both inoculated, unvaccinated gilts were first detected positive for CSF on VI in whole blood 6-days post-

\begin{tabular}{llccccccc}
\hline & \multicolumn{2}{l}{ Seeders $(n=6)$} & \multicolumn{2}{c}{ Contacts $1(n=15)$} & \multicolumn{2}{c}{ Contacts $2(n=10)$} \\
\cline { 2 - 8 } $\begin{array}{l}\text { Days post- } \\
\text { inoculation }\end{array}$ & VI & RT-nPCR & VI & RT-nPCR & Diff. ELISA $^{\mathrm{b}}$ & VI & RT-nPCR & VN $^{\text {c }}$ \\
\hline 0 & $0^{\mathrm{a}}$ & 0 & 0 & 0 & 0 & - & - & - \\
2 & 0 & 4 & 0 & 0 & 0 & - & - & - \\
4 & 5 & 6 & 0 & 0 & 0 & - & - & - \\
6 & 6 & 6 & 0 & 0 & 0 & - & - & - \\
8 & 6 & 6 & 0 & 0 & 0 & - & - & - \\
10 & 6 & 6 & 0 & 0 & 0 & - & - & - \\
12 & 6 & 6 & 0 & 0 & 0 & - & - & - \\
14 & 6 & 6 & 0 & 0 & 0 & - & - & - \\
16 & 6 & 6 & 0 & 2 & 0 & - & - & - \\
18 & - & - & 0 & 1 & 0 & 0 & 0 & 0 \\
20 & - & - & 0 & 0 & 1 & 0 & 0 & 0 \\
22 & - & - & 0 & 2 & 1 & 0 & 0 & 0 \\
24 & - & - & 0 & 0 & 2 & 0 & 0 & 0 \\
26 & - & - & 0 & 0 & 6 & 0 & 0 & 0 \\
28 & - & - & 0 & 0 & 8 & 0 & 0 & 0 \\
30 & - & - & 0 & 0 & 10 & 0 & 0 & 0 \\
32 & - & - & 0 & 0 & 11 & 0 & 0 & 0 \\
34 & - & - & 0 & 0 & 13 & 0 & 0 & 0 \\
36 & - & - & 0 & 0 & 13 & 0 & 0 & 0 \\
41 & - & - & 0 & 0 & 13 & 0 & 0 & 0 \\
48 & - & - & 0 & 0 & 13 & 0 & 0 & 0 \\
\hline
\end{tabular}

${ }^{\mathrm{a}}$ The number of positive animals.

${ }^{\mathrm{b}}$ Diff. ELISA, differential ELISA.

${ }^{\mathrm{c}} \mathrm{VN}$, virus neutralization. 
Table 2. Results of experiment 3

\begin{tabular}{lccccc}
\hline & \multicolumn{2}{c}{ Seeders $(n=2)$} & \multicolumn{3}{c}{ Contacts $(n=8)$} \\
\cline { 2 - 6 } $\begin{array}{l}\text { Days post- } \\
\text { inoculation (pi) }\end{array}$ & VI & RT-nPCR & VI & RT-nPCR & Diff. ELISA \\
\hline 0 & $0^{\text {a }}$ & 0 & 0 & 0 & 0 \\
3 & 0 & 1 & 0 & 0 & 0 \\
6 & 2 & 2 & 0 & 0 & 0 \\
9 & 2 & 2 & 0 & 2 & 0 \\
12 & 2 & 2 & 0 & 7 & 0 \\
15 & 2 & 2 & 0 & 6 & 0 \\
18 & 2 & 2 & 0 & 0 & 0 \\
21 & $1^{\mathrm{b}}$ & $1^{\mathrm{b}}$ & 0 & 0 & 2 \\
24 & - & - & 0 & 0 & 3 \\
27 & - & - & 0 & 0 & 5 \\
30 & - & - & 0 & 0 & 8 \\
33 & - & - & 0 & 0 & 8 \\
36 & - & - & 0 & 0 & 8 \\
39 & - & - & 0 & 0 & 8 \\
42 & - & - & 0 & 0 & 8 \\
49 & - & - & 0 & 0 & 8 \\
56 & - & - & 0 & 0 & 8 \\
61 & - & - & 0 & 0 & 8 \\
70 & - & - & 0 & 0 & 8 \\
\hline
\end{tabular}

${ }^{\mathrm{a}}$ The number of positive animals.

${ }^{\mathrm{b}}$ At day 19 pi one seeder pig died, the second died at day 21 pi.

inoculation (dpi). These gilts also developed typical clinical symptoms and high fever. Eventually, both gilts died between 19- and 21-dpi.

In the vaccinated contact animals, no viraemia was detectable using VI. Using RT-nPCR, positive blood samples were found in seven of eight pregnant contact gilts. For those samples that were positive in RT-nPCR but remained negative in VI, three additional cell-culture passages were performed. All remained negative.

All eight pregnant vaccinated contact gilts became positive in the differential ELISA.

Of the seven RT-nPCR positive vaccinated pregnant gilts, three had CSFV infected offspring. In the infected piglets, virus was detectable using VI. None of the infected offspring had seroconverted against the CSFV.

\section{Discussion}

In previous experiments (Dewulf et al., 2000, 2002), we have observed that E2 sub-unit marker vaccine vaccinated pigs can become infected and may react positive in the RT-nPCR test without the possibility to detect the virus using the conventional VI technique. In experiment 2, we were able to reproduce this phenomenon indicating that this was not a coincidental observation. Moreover, it was observed in the previous and current experiments that the RT-nPCR positive pigs also developed antibodies against the wild-type virus indicating that they were really infected.

It has been demonstrated that in unvaccinated CSF infected pigs, RT-nPCR becomes positive significantly earlier than the conventional VI techniques (Paton et al., 2000; Dewulf et al., 2004). This is explained by the fact that using RT-nPCR, it becomes possible to detect very small virus doses that are below the detection limit of the conventional diagnostic techniques such as VI. However, RT-nPCR has the drawback that if a positive result is obtained, it is not unequivocally linked to the presence of an infectious particle in the sample because of the fact that it only amplifies a specific genomic region (Hilfenhaus et al., 1997). Therefore, a RT-nPCR may be positive because of the detection of pieces of RNA from disintegrated viruses or because of the detection of harmless complexes of antigens and antibodies.

As a result, it remains unclear whether these VI negative and RT-nPCR positive animals are infectious or not? The answer to this question is also important in the light of the fact that it has been described that virus transmission by direct contact from E2 sub-unit marker-vaccine vaccinated pigs, inoculated with CSFV, towards sentinel pigs has occurred occasionally although these vaccinated pigs did not develop a detectable viraemia in VI (Bouma et al., 1999). Whether these pigs were positive in the RT-nPCR test was not reported.

In the first experiment, no effect was seen in pigs inoculated with serum originating from vaccinated RT-nPCR pigs, indicating that the serum did not contain enough viable CSF virus to infect the sentinels. Although, it is unlikely, this result might also be due to freezing and storage of the serum.

To avoid this, in the second experiment, RT-nPCR positives were directly brought in contact with susceptible pigs. This set up had the additional advantage that even pigs with a very short viraemic period that might be missed because of the sampling interval, had the opportunity to transmit the virus if they were infectious. Although 12 of the 15 contact pigs $\left(C_{1}\right)$ became infected (positive in the differential ELISA) and three of 15 contact pigs were positive in the RT-nPCR test while they were housed together with the sentinel $\mathrm{C}_{2}$ contact pigs (one was viraemic just before being moved to the sentinels), none of these $\mathrm{C}_{2}$ pigs became infected. As a result, also in this experiment no proof of horizontal virus transmission from vaccinated RT-nPCR positive pigs to sentinel pigs could be observed.

In the third experiment, again a number of the vaccinated animals became positive in the RT-nPCR test. In three of seven RT-nPCR positive and pregnant sows, a transplacental infection of the foetuses was observed. Consequentially, in this experiment a clear proof of vertical virus transmission was found in naturally infected vaccinated pregnant gilts that had a viraemia, which was only detectable using RT-nPCR. In this experiment, the occurrence of horizontal virus transmission could not be evaluated. In this experiment, it should be added that blood samples of the vaccinated gilts were only taken on a 3-days interval and therefore there is an increased probability that an episode of a VI positive viraemia could be missed. In the previous two experiments, blood samples were taken every other day.

The observed difference in the probability of horizontal and vertical transmission may be explained by the fact that foetuses are continuously exposed to the virus during the period of viraemia whereas pen mates are only exposed intermittently. Another explanation might be the fact that fewer infectious viruses are necessary to infect a piglet transplacentaly than oronasaly. Finally, it is possible that the CSFV is hidden from the neutralizing antibodies by surviving in the white blood cells (WBC). Protected by these WBC, the virus might migrate through the placental barrier whereas no WBC containing CSFV are normally excreted, and free virus is probably neutralized by $\operatorname{IgA}$ in the mucosal membranes.

Based on these findings, it can be concluded that if a vaccinated pig is positive in RT-nPCR but negative in VI the level of circulating virus might still be high enough for vertical 
virus transmission, but is probably not high enough for horizontal virus transmission.

\section{Acknowledgements}

This study was supported by grants from the Research Foundation of the Belgian Ministry of Public Health and the Fund for Health and Quality of Animals and Derived Products.

\section{References}

Bouma, A., A. J. de Smit, E. P. de Kluijver, C. Terpstra, and R. J. Moormann, 1999: Efficacy and stability of a subunit vaccine based on glycoprotein E2 of classical swine fever virus. Vet. Microbiol. 66, 101-114.

Dewulf, J., H. Laevens, F. Koenen, H. Vanderhallen, K. Mintiens, H. Deluyker, and A. de Kruif, 2000: An experimental infection with classical swine fever in E2 sub-unit marker-vaccine vaccinated and in non-vaccinated pigs. Vaccine 19, 475-482.

Dewulf, J., H. Laevens, F. Koenen, K. Mintiens, and A. de Kruif, 2002: An E2 sub-unit marker-vaccine does not prevent horizontal or vertical transmission of classical swine fever. Vaccine 20, 86-91.

Dewulf, J., F. Koenen, K. Mintiens, P. Denis, S. Ribbens, and A. de Kruif, 2004: Analytical performance of several classical swine fever diagnostic techniques on live animals for the detection of infection. J. Virol. Methods 119, 137-143.

Floegel-Niesmann, G., C. Bunzenthal, S. Fischer, and V. Moennig, 2003: Virulence of recent and former classical swine fever isolates evaluated by their clinical and pathological signs. J. Vet. Med. B 50, 214-220.

Hilfenhaus, J., A. Groner, T. Nowak, and T. Weimer, 1997: Analysis of human plasma products: polymerase chain reaction does not discriminate between live and inactivated viruses. Transfusion 37, 935-940.
Holm-Jensen, M., 1981: Detection of antibodies against hog cholera virus and bovine viral diarrhoea in porcine serum. A comparative examination using CF, PLA, and NPLA assay. Acta Vet. Scan. 22, 85-98.

Hulst, M. M., D. F. Westra, G. Wensvoort, and R. J. Moormann, 1993: Glycoprotein E1 of hog cholera virus expressed in insect cells protects swine from hog cholera. J. Virol. 67, 5435-5442.

Koenen, F. and J. Lefebvre, 1994: Kinetics of an experimental infection with a classical swine fever (CSF) field isolate. In: Schwyzer, M, M. Ackermann, G. Bertoni, R. Kocherhans, K. Mc Culloch, M. Engels, R. Wittek, and R. Zanoni (eds), Proc. Third Congress European Society of Veterinary Virology, pp. 322-326. Interlacken, Switzerland.

Koenen, F., G. Van Caenegem, J. P. Vermeersch, J. Vandenheede, and H. Deluyker, 1996: Epidemiological characteristics of an outbreak of classical swine fever in an area of high pig density. Vet. Rec. 139, 367-371.

McGoldrick, A., E. Bensaude, G. Ibata, G. Sharp, and D. J. Paton, 1999: Closed one-tube reverse transcription nested polymerase chain reaction for the detection of pestiviral RNA with fluorescent probes. J. Virol. Methods 1, 85-95.

Meuwissen, M. P., S. H. Horst, R. B. Huirne, and A. A. Dijkhuizen, 1999: A model to estimate the financial consequences of classical swine fever outbreaks: principles and outcomes. Prev. Vet. Med. 42, 249-270.

Moormann, R. J., A. Bouma, J. A. Kramps, C. Terpstra, and H. J. de Smit, 2000: Development of a classical swine fever subunit marker vaccine and companion diagnostic test. Vet. Microbiol. 73, 209-219.

Paton, D. J., A. McGoldrick, S. Belak, C. Mittelholzer, F. Koenen, H. Vanderhallen, M. Biagetti, G. De Mia, T. Stadejek, M. A. Hofmann, and Thuer B. 2000: Classical swine fever virus: a ring test to evaluate RT-PCR detection methods. Vet. Microbiol. 73, 159174.

Terpstra, C., 1998: Preventive emptying: a compensation for a lack of training. Tijdschr. Diergeneeskd. 123, 324-325. 